Pathologe 2010 · [Suppl 2] 31:318-319

DOI 10.1007/s00292-010-1314-1

Online publiziert: 16 . Juli 2010

(c) Springer-Verlag 2010

\author{
R. Knüchel-Clarke' ${ }^{1}$ A. Hartmann ${ }^{2}$ \\ ${ }^{1}$ Institut für Pathologie, Medizinische Fakultät RWTH Aachen, \\ Universitätsklinikum Aachen \\ ${ }_{2}^{2}$ Pathologisches Institut, Universitätsklinikum Erlangen
}

\title{
Sitzungsbericht der AG Uropathologie
}

\section{Aktivitäten aus dem vergangenen Jahr}

Die avisierten Bemühungen um den Kontakt zur Deutschen Gesellschaft für Urologie (DGU) wurden erfolgreich umgesetzt. Das Ergebnis ist ein Programmpunkt bei dem diesjährigen Kongress der DGU in Düsseldorf. Es wird eine Forumssitzung Uropathologie am 25. September 2010 von 10.30 bis 12.00 Uhr unter Beteiligung von 4 Pathologen interdisziplinär mit Urologen zu den 4 Organtumoren der Uroonkologie stattfinden.

Auf Einladung des Berufsverbandes Pathologie und nach Rücksprache mit Prof. Dietel als Präsident der Deutschen Gesellschaft für Pathologie (DGP) hat Frau Knüchel-Clarke eine 4-stündige Sitzung zum Thema Prostatakarzinom geleitet. Dieses erfolgte in Zusammenarbeit mit Prof. Heidenreich, Direktor der Urologischen Klinik, RWTH Aachen. Es wurden die Leitlinien der europäischen und deutschen Urologen vorgestellt, und es folgte eine Darstellung und Diskussion zu den Themen DNA-Zytometrie der Prostata (Prof. Böcking und Prof. Helpap) und ein Beitrag zur molekularen Pathologie von Prof. Hartmann.

\section{Neue Inititiativen der Arbeitsgemeinschaft}

Für die weitere Struktur der AG-Sitzung wird ein Themenschwerpunkt mit Gastvortrag für die Sitzung 2011 vorgeschlagen. Die zukünftige inhaltliche Gestaltung könnte derart erfolgen, dass ein inhaltlicher Schwerpunkt mit Gastred- ner $1 / 3$ oder $1 / 2$ der Sitzungszeit der AG ausmacht und die anderen Themen ergänzend behandelt werden. Es wird von Herrn Sauter betont, dass es das Ziel sein müsse, eine hohe Attraktivität der Veranstaltung zu erreichen. Erste Vorschläge für einen Themenschwerpunkt sind Leitlinien, Zystenniere (Pädiatrie, Nephrologie), Bericht eines Urologen zur Forschungskonzeption und Tumorbankinitiativen der DGU und des Bundesministeriums für Bildung und Forschung (BMBF). Der Tatsache, einen Schwerpunkt innerhalb der AG-Sitzung zu setzen, wird allgemein zugestimmt. Es wird zusammen mit dem Satzungsvorschlag eine Rundfrage zum Schwerpunktthema 2011 erfolgen. Prof. Moch betont als Geschäftsführer der DGP, dass die Zeit für die Arbeitsgemeinschaften nach Aktivität und Aktualität ausgerichtet werden können.

\section{Inhaltliche Definition der Arbeitsgemeinschaft}

Die Diskussion zum Thema Uropathologie mit Nephropathologie wird erneut geführt. Die Diskussion lohnt sich aus der Erfahrung von Herrn Moch deswegen, weil sich eine neue AG Gefäßpathologie gegründet hat, die für einen Teil der nephrologischen Beiträge geeignet sei. Von Seiten der Nephrologie (Dr. Becker) wird betont, dass die Gemeinsamkeit mit der Uropathologie wichtig sei, weil z. B. sein Arbeitsbereich Nephrologie und Uropathologie umfassen würde. Frau KnüchelClarke betont, dass nur ein Teil der nephrologischen Beiträge inhaltlich den kardiovaskulären Bereich betrifft, diese könnten aktiv der AG Gefäßpathologie zugeordnet werden, während andere nephrologische Themen für den Austausch mit den uropathologisch Tätigen sinnvoll seien.

Als Fazit ist festzuhalten, dass die Situation im Kontext mit dem kommenden oder den kommenden Kongress(en) bewertet werden soll und vorab die Gemeinsamkeit gesucht wird.

Des Weiteren werden sich die Mitglieder der AG um die Formulierung einer Basissatzung kümmern und die Mitglieder spätestens vor der DGP-Tagung, wenn sinnvoll auch zwischenzeitlich, informieren. Neue Mitglieder sind weiterhin willkommen.

\section{Wissenschaftlicher Teil der Sitzung}

Nach Entnahme von Beiträgen für das Hauptprogramm betrug die Anzahl der uropathologischen und nephrologischen Beiträge noch mehr als 40. Diese wurden unter Berücksichtigung verschiedener Themenschwerpunkte und in Abhängigkeit von der Qualität in die Sitzung der AG integriert oder ebenfalls nach Themen gruppiert verschiedenen Poster-Sessions zugeordnet.

Im ersten Teil der Sitzung waren die Themen Nephrologie, Nierentumor und Harnblasenkarzinom (Abstract Do-06o bis Do-071 [1]). Im Bereich der Nephropathologie wurden zunächst durch die Arbeitsgruppe aus der Abteilung für zelluläre molekulare Pathologie des Deutschen Krebsforschungszentrums (DKFZ) in Heidelberg Arbeiten zur zerebralen thrombotischen Mikroangiopathie vor- 
gestellt. Dabei konnte durch Porubsky et al. gezeigt werden, dass eine tubuläre Defizienz von Gb3 zwar das durch Verotoxin vermittelte Nierenversagen, jedoch nicht eine zerebrale thrombotische Mikroangiopathie verhindert.

Die Arbeitsgruppe vom Institut für $\mathrm{Pa}$ thologie der Universität Heidelberg (Koleganova et al.) zeigte dann in zwei Vorträgen experimentelle Arbeiten zum Einfluss von Erythropoetin sowohl auf die Niereninsuffizienz als auch auf die experimentelle urämische Kardiomyopathie.

Mehrere Arbeiten beschäftigten sich mit molekularen Veränderungen bei der Entstehung des Nierenzellkarzinoms. In einer umfangreichen Untersuchung zeigte die Arbeitsgruppe aus dem Institut für Pathologie der Universität Hamburg (Minner et al.) Ergebnisse zur Bedeutung von Amplifikationen und Mutationen von EGFR in Nierentumoren. Weitere Arbeiten beschäftigten sich mit der Bedeutung verschiedener Mutationen im VHLGen für die Proteinfunktion in den sporadischen Nierenzellkarzinomen und mit der molekular-epidemiologischen Untersuchung zur Bedeutung eines Polymorphismus im p53-Gen $\left(R_{72} P\right)$ für das Risiko eines Nierenzellkarzinoms und dessen Verlauf.

Schließlich wurde in 4 Beiträgen zum Harnblasenkarzinom der prädiktive Wert der Expression von MDR1 und ERCC 1 bei Patienten mit fortgeschrittenen Harnblasenkarzinomen und adjuvanter Therapie untersucht. Hier konnte durch Analyse des retrospektiv gesammelten Materials einer großen prospektiven Studie gezeigt werden, dass die Expression beider Proteine einen wesentlichen Einfluss auf den Erfolg der adjuvanten Chemotherapie hat.

In den letzten beiden Vorträgen wurden durch die Arbeitsgruppe aus dem Institut für Pathologie in Aachen erste Ergebnisse von Proteomanalysen beim Harnblasenkarzinom gezeigt. Hier konnte durch Henkel et al. und Oezdemir et al. gezeigt werden, dass in Harnblasenkarzinomen unterschiedlicher Differenzierung differenziell exprimierte Proteine identifiziert werden können.

Der zweite Teil der Sitzung war durch die Prostatakarzinomforschung bestimmt (Abstract Do-072 bis Do-08o [1]). Die Präsentationen gaben Einblick in neue
Techniken der Metabolomforschung, die als mehrparametrischer Ansatz in Analogie zur vorab an Urothel dargestellten Proteomforschung massenspektrometrische Messungen an Gefriermaterial erlaubt, an denen die Unterscheidung von Tumorund Normalgewebe gelingt (Kristiansen et al., Zürich). Die Tübinger Pathologie berichtete über neue Daten zum ERG-Rearrangement, die im Rahmen internationaler Kooperationen erhoben wurden. Insbesondere die Prostataspezifität des Rearrangements wurde in einer großen Serie an gängigenTumoren belegt (Scheble et al., Tübingen).

Verschiedene zellbiologische Arbeiten wurden vorgestellt, die Signalwege in der Prostata auf therapeutische Ansätze hin untersuchten. Hierbei wurde beispielsweise auch ein sehr umfangreicher RNAi-Screen verwendet, um letale Interaktionen mit dem PTEN-Gen zu identifizieren. Dabei werden synthetische Moleküle identifiziert, die in einer Zelle mit „PTEN knock-down“ zur vermehrten Apoptose und zum Zelltod führen (Wild et al., Zürich).

Abschließend wurden durch einen Kollegen aus der Urologie und durch Prof. Helpap klinisch-praktische Probleme wie die Aufarbeitung des Prostatektomiepräparates und die Zweitmeinungskultur zur Diskussion gebracht.

\section{Korrespondenzadresse \\ Prof. Dr. R. Knüchel-Clarke}

Institut für Pathologie, Medizinische Fakultät RWTH Aachen, Universitätsklinikum Aachen Pauwelsstr. 30, 52074 Aachen

rknuechel-clarke@ukaachen.de

Interessenkonflikt. Die korrespondierende Autorin gibt an, dass kein Interessenkonflikt besteht.

\section{Literatur}

1. 94. Jahrestagung der Deutschen Gesellschaft für Pathologie e.V. (2010) Der Pathologe, 3. Sonderheft, Abstract Do-060-080, 19-24 